\title{
Robust Nonsingularity Analysis Using Linear Fractional Transformation and Its Application to Stability Analysis
}

\author{
Chwan-Lu Tseng and I-Kong Fong \\ Department of Electrical Engineering \\ National Taiwan University \\ Taipei, Taiwan 10617, Republic of China
}

\begin{abstract}
In this paper, a procedure is described for converting a perturbed matrix with mixed unstructured and structured uncertainties to the form of a Linear Fractional Transformation (LFT). Also, a criterion is given for keeping the nonsingularity of the perturbed matrix, provided it is nominally so. Moreover, based on the conversion method and criterion, explicit bounds on the time-varying uncertainties in continuous-time and discrete-time systems are derived for robust stability.
\end{abstract}

\section{Introduction}

Recently, matrix nonsingularity analysis has been utilized to analyze the robust stability problem in the presence of time-invariant/varying uncertainty $[1,2]$. However, the dimensions of the matrices involved tend to be large, especially in the approaches involving Kronecker product and structured singular value $(\mu)$ analysis techniques, such as in [2]. This may cause some computation problems. Moreover, all these approaches can deal with multivariable polynomial type of uncertainties at most [1]. In this paper, we introduce a method to deal with the robust nonsingularity problem. It is shown how to convert a matrix with mixed unstructured and bounded rational type structured uncertainties to the form of an LFT. Then, for the case in which the nominal matrix is nonsingular, an explicit bound is given in terms of $\mu$ for the perturbed matrix to remain nonsingular. Compared with previous results in this respect, the current method can deal with a wider variety of uncertainties, including even the product of unstructured and structured uncertainties. In addition, the dimensions of the matrices involved now are often smaller than those from the Kronecker product approach. Furthermore, the derived result is still applicable to the robust stability analysis problems, and bounds of uncertainties for the uncertain systems to preserve stability can be obtained by using the concept of nonsingularity check. Thus a wider class of the robust stability analysis problems can be solved with the proposed method in a more efficient way.

\section{Robust Nonsingularity Analysis Using LFT Representation}

Let $k=\left[\begin{array}{lll}k_{1} & \cdots & k_{q}\end{array}\right]^{T} \in \mathbf{R}^{q}$ be a vector of bounded uncertain parameters, $\Delta_{F i} \in \mathbf{R}^{l_{i} \times l_{i}}, i=1, \ldots, p$, be bounded unstructured uncertainty matrices, $\Delta=\operatorname{diag}\left\{k_{1} I_{r_{1}}, \ldots\right.$, $\left.k_{q} I_{r_{q}}, \Delta_{F_{1}}, \ldots, \Delta_{F_{p}}\right\}$, where $r_{i}$ is the multiplicity of $k_{i}$, and $\Omega_{\gamma}=\{\Delta: \bar{\sigma}(\Delta)<\gamma\}$, where $\left.\sigma \overline{(} \cdot\right)$ is the largest singular value. Then we have

Definition 2.1 It is said that a perturbed matrix $S \in \mathbf{R}^{n \times n}$ containing $k_{1}, \ldots, k_{q}$ and $\Delta_{F_{1}}, \ldots, \Delta_{F_{p}}$ can be transformed to an LFT $F(M, \Delta)$ on $\Delta$ if $S$ can be written as

$$
\begin{aligned}
S & =M_{22}+M_{21}\left(I_{r}-\Delta M_{11}\right)^{-1} \Delta M_{12} \\
& \triangleq F(M, \Delta),
\end{aligned}
$$

where $M_{11}, M_{12}, M_{21}$, and $M_{22}$ are constant matrices of suitable dimensions, $M$ is the joint coefficient matrix defined as $\left[\begin{array}{l|l}M_{11} & M_{12} \\ \hline M_{21} & M_{22}\end{array}\right]$, and $r=\sum_{i=1}^{q} r_{i}+\sum_{i=1}^{p} l_{i}$.

From the definition, it is easily seen that $M_{22}$ equals to the nominal part of $S$. Moreover, if $\operatorname{det}\left(I_{r}-\Delta M_{11}\right) \neq 0$ for all $\Delta \in \Omega_{\gamma}$, then the LFT is said to be well-defined over $\Omega_{\gamma}$. Now, suppose the perturbed matrix $S$ considered in this paper is of the following form:

$$
S=S_{o}+S_{s}(k)+S_{u},
$$

where $S_{o}+S_{s}(0)$ is the nominal part of $S$ and assumed to be nonsingular. $S_{s}(k)-S_{s}(0)$ is the structured part of the perturbation, and $S_{u}$ is the unstructured part of the perturbation. Assume further $S_{s}(k)$ has bounded rational entries $f_{i j}(k)$ of the form

$$
f_{i j}(k)=\frac{n_{i j}(k)}{1+d_{i j}(k)},
$$

where $n_{i j}(k)$ and $d_{i j}(k)$ are multivariable polynomials in the elements of $k$, and $1+d_{i j}(k) \neq 0 \forall k \in \Omega_{\gamma}$. Moreover, $S_{u}$ is of the form

$$
S_{u}=D_{1} \Delta_{F} E_{1} G_{\Delta}
$$

where $D_{1}$ and $E_{1}$ are constant matrices, $\Delta_{F}=\operatorname{diag}\left\{\Delta_{F_{1}}, \ldots\right.$ , $\left.\Delta_{F_{p}}\right\}$, and $G_{\Delta}$ has the meaning explained as follows. If $S_{u}$ is a linear function of $\Delta_{F}$ only, then $G_{\Delta}=I$, i.e., $S_{u}=D_{1} \Delta_{F} E_{1}$. If $S_{u}$ is the quadratically-coupled unstructured uncertainty, then we can set $G_{\Delta}=\Delta_{F}^{\prime} E_{2}$, where $E_{2}$ is a constant matrix, and $\Delta_{F}^{\prime}=\operatorname{diag}\left\{\Delta_{F_{p+1}}, \ldots, \Delta_{F_{p}}\right\}$. Finally, if $S_{u}$ is the product of structured and unstructured uncertainties, then we can set $G_{\Delta}=G(k)$, where $G(k)$ is a matrix of the bounded rational entries, just the same as $S_{s}(k)$.

With the techniques proposed in [6], it is known that $f_{i j}(k)$ can always be converted to an LFT. As a result, by simply adding the $(i, j)$ th entry of $S_{o}$ to $f_{i j}(0)$, the $(i, j)$ th entry of $S_{o}+S_{s}(k)$ can be converted to an LFT $F\left(M^{i j}, \Delta^{i j}(k)\right)$, where $M^{i j}=\left[\begin{array}{c|c}M_{11}^{i j} & M_{12}^{i j} \\ \hline M_{21}^{i j} & M_{22}^{i j}\end{array}\right]$, and $\Delta^{i j}(k)$ is a diagonal matrix with elements of $k$ repeated on the diagonal. After transforming the entries of $S_{o}+S_{s}(k)$ to LFTs individually, we have

$$
S_{o}+S_{s}(k)=\bar{M}_{22}+\bar{M}_{21}\left[I_{d}-\bar{\Delta}(k) \bar{M}_{11}\right]^{-1} \bar{\Delta}(k) \bar{M}_{12}
$$

where

$$
\begin{aligned}
& \bar{M}_{11}=\operatorname{diag}\left\{M_{11}^{11}, \ldots, M_{11}^{1 n}, \ldots, M_{11}^{n 1}, \ldots, M_{11}^{n n}\right\} \\
& \bar{M}_{12}=\left[\begin{array}{c}
\operatorname{diag}\left\{M_{12}^{11}, \ldots, M_{12}^{1 n}\right\} \\
\ldots \\
\operatorname{diag}\left\{M_{12}^{n 1}, \ldots, M_{12}^{n n}\right\}
\end{array}\right] \\
& \bar{M}_{21}=\operatorname{diag}\left\{M_{21}^{(1)}, \ldots, M_{21}^{(n)}\right\}
\end{aligned}
$$


with $M_{21}^{(j)}=\left[M_{21}^{j 1} M_{21}^{j 2} \cdots M_{21}^{j n}\right], \bar{M}_{22}$ is a block matrix with the $(i, j)$ th block $M_{22}^{i j}, \bar{\Delta}(k)=\operatorname{diag}\left\{\Delta^{11}(k), \ldots, \Delta^{1 n}(k), \ldots\right.$ $\left., \Delta^{n}(k), \ldots, \Delta^{n n}(k)\right\}$, and $I_{d}$ is the identity matrix with the same dimension as that of $\bar{\Delta}(k)$. After interchanging rows and columns of $\bar{\Delta}(k)$ so that the elements of $k$ appear in order, the procedure discussed in [6] can be used to reduce the size of the uncertainty matrix.

Next we turn to the unstructured part. If $G_{\Delta}$ falls in any of the above-mentioned categories of uncertainty, it is obviously an LFT, or can be transformed to one. Thus, $S_{u}$ can be converted to an LFT because the product of two LFTs can always be converted to another LFT. Therefore, since the sum of LFTs can be converted to still another LFT, we reach at the conclusion that $S$ can be expressed as an LFT. In fact, in addition to the aforementioned categories of $G_{\Delta}$, at this point it is easy to see that the same conclusion holds for any $G_{\Delta}$ that is an LFT itself, or one that is obtained by using the procedure recursively. Without loss of generality, we assume that $S$ can be written as

$$
S=M_{22}+M_{21}\left(I_{r}-\Delta M_{11}\right)^{-1} \Delta M_{12}, \quad \Delta \in \Omega_{\gamma},
$$

where $\operatorname{det} M_{22} \neq 0$. Thus, the remaining question is how large $\gamma$ can be to keep $S$ (in form of an LFT) nonsingular.

Theorem $1([3,4])$ The LFT of $S$ is well-defined and $S$ keeps nonsingularity over $\Omega_{\gamma}$ if and only if

$$
\gamma \leq 1 / \max \left\{\mu\left(M_{11}\right), \mu\left(M_{11}-M_{12} M_{22}^{-1} M_{21}\right)\right\}
$$

where $\mu(\cdot)$ is the structured singular value [5] with respect to the set of real matrices of the form $\Delta$.

From the above theorem and the conversion techniques introduced, it is clear that the robust nonsingularity problems which can be dealt with by the method of this paper is more general than those in $[1,2]$. It is also noted that the sizes of resulting matrices are often smaller than that obtained by using Kronecker prodluct (if possible) from numerical experiences.

\section{Robust Stability Analysis}

Consider linear uncertain systems of order $n$ with the state-space model

$$
\begin{aligned}
\delta x(t) & =A(k(t)) x(t) \\
& =\left[A_{0}+\sum_{i=1}^{m} f_{i}(k(t)) A_{i}\right] x(t),
\end{aligned}
$$

where $\delta$ denotes the differentiation operator for continuoustime systems, or the shift operator for discrete-time systems, $x(t) \in \mathbf{R}^{n}$ is the state vector, $k(t)$ is the vector of $q$ time-varying uncertain parameters, $f_{i}$ 's are bounded rational functions similar to that in eq. (4) but $f_{i}(0)=0$, and $A_{i}$ is the structural influence matrix through which $f_{i}$ affects $A(k(t))$. Moreover, it is assumed that $A_{0}$ is stable The argument $t$ will be omitted if no ambiguities arise in the following discussion.

Define the Lyalunov function candidate by $V(x)=$ $x^{T} P x$, where $P$ satisfies

$$
A_{0}^{T} P^{P}+P A_{0}=-Q
$$

for the continuous-time case and

$$
A_{0}^{T} P A_{0}+P=-Q
$$

for discrete-time case, and $Q$ is positive definite. Thus, the Lyapunov derivative (difference) $\mathcal{L}(V(x))$ becomes

$$
\begin{aligned}
\mathcal{L}(V(x)) & =x^{T} L(k) x \\
& =x^{T} F(M, \Delta(k)) x
\end{aligned}
$$

where $L(k)=-Q+\sum_{i=1}^{m} f_{i}(k)\left(A_{i}^{T} P+P A_{i}\right)$ for continuoustime systems and $L(k)=-Q+\sum_{i=1}^{m} f_{i}(k)\left(A_{0}^{T} P A_{i}+A_{i}^{T} P A_{0}\right.$ )$+\sum_{i, j=1}^{m} f_{i}(k) f_{j}(k) A_{i}^{T} P A_{j}$ for discrete-time systems. Note that for either systems $L(k)$ has bounded rational entries, and hence we can rewrite $L(k)$ as $F(M, \Delta(k))$. Since $M_{22}=$ $-Q$, by using the concept of robust nonsingularity [2], the following robust stability theorem can be obtained.

Theorem 2 System (9) is asymutotically stable if

$$
\|k\|_{\infty}<\left\{\max \left\{\mu\left(M_{11}\right), \mu\left(M_{11}-M_{12} M_{22}^{-1} M_{21}\right)\right\}\right\}^{-1} .
$$

From the above theorem, it is clear that the proposed method can deal witlı a wider class of uncertainties than those in $[1,2,7,8]$. Furthermosre, for a given $Q$, the derived bound is maximal to ensure the negativeness of $\mathcal{L}(V(x))$ in the sense of $l_{\infty}$ norm. However, it is noted that different choices of $Q$ may affect the bound found.

\section{Conclusion}

In this paper, we develop an LFT approach to solve the robust nonsingularity problem via $k$-allalysis. It is shown that the proposed method can deal with a wider variety of uncertainties, such as the mixed unstructured and/or rational type structured uncertainties, and the obtained uncertainty matrix is often of smaller size. Also, an application in robust stability analysis is given to show the usefulness of the method.

\section{References}

[1] A. Tesi and A. Vicino, "Robust Stability of StateSpace Models with Structured Uncertainties," IEEE Trans. Automat. Contiol, Vol. A(:-35, No. 2. pp. 191195, Feb., 1990.

[2] C.-L. Tseng, J-K. Fong, and J.-H, Su, "Stalsility Analysis of Systems with Structured Time-Varying Uncertainties Using Structured Singular Value, presented in the Brol SIAM Conf. on Linear Algobra in Signals, Systems and Control, Seat, le, Washington, August, 1993

[3] A. K. Parkard, What is Ncw With $\mu$ : Structured Uncerlainly in Mullivariable Control, Ph. D thesis, University of Califormia, Berkeley, 1988.

[4] C.-L. Tseng and I-K. Fong "Robust Nonsingularity Analysis Using Lincar Rardional Thusslormation," Proc. 1994 R. O. C. Automatic Control Confrerner, Longtang, Taiwan, pp). $281-284$, March, 1994.

[5] J. C. Doyle, "Analysis of Foedhack Systems with Structured Uncertainties," IEE P'rocedding.s, Vol. J2!), Part D, No. 6, pp. 2.12-250, Nov., 1982.

[6] P. Lambrechts, J. Terlonw, S. Bennani, and M Steinbuch, "Parametric Incertainty Modeling Using LPTs," Proc. American Contr. Conf., San Francisco, California, pp. 267-272, June, 19993.

[7] II. A. Latchman and .J. A. Letra, "On the Computation of Allowalse [Bondeds for Parametric Incertainty," Proe. American Contr. Conf., Boston, Massarchusetts, p). 867-86s, June, 199!1.

[8] J.-II. Su and I-K. Fong, " Rolsust Stahility Analy sis of Linear Continuous/Discrete-Time Systems with Output Feedback Controllers," IEEE Trans. A ulomat. Control, Vol. AC-38, No. 7 , p1. 1154-1158, July, 1993. 